\title{
Technology Enhanced Interaction Framework and Method for Accessibility in Thai Museums
}

\author{
Kewalin Angkananon, Mike Wald, Lester Gilbert \\ Electronic and Computer Science \\ University of Southampton \\ Southampton, UK \\ ka3e10@ecs.soton.ac.uk
}

\begin{abstract}
People with hearing impairments have difficulties in their access to Thai in situ authentic culture because they have problems with understanding spoken information during their visit. An extensive review of the literature found that there was no framework to guide developers in developing technology solutions for such face-to-face situations for people with hearing impairment, other disabilities or no disabilities. This paper describes a Technology Enhanced Interaction Framework (TEIF) and Method that have been shown by experiments and questionnaires to have the potential to help developers make local Thai museums more accessible for disabled visitors. The mean number of correct requirements was significantly higher for participants using the TEIF Method than the Other Methods. The TEIF Method helped developers select a solution significantly more often than the Other Methods that was not worse than a solution rated best by the experts. The TEIF Method also helped developers rate the best solution significantly closer to the experts' ratings than the Other Methods.
\end{abstract}

Keywords-museums; framework; method; accessibility; technology

\section{INTRODUCTION}

Thai culture is changing over time because of exposure to other nationalities whether face to face or through the Internet, television, or films. Small local Thai museums run by their owners help preserve Thai everyday culture in its original position or place by passing on knowledge and wisdom. Visiting such local museums is more realistic and authentic compared to an experience inside a large museum but there is less budget support from the government [1] so owners of such local sites often do not have enough funding to provide accessible information for hearing impaired people. People with hearing impairments have difficulties in their access to Thai in situ authentic culture because they have problems with understanding spoken or complex text information during their visit. There is more research into helping people with hearing impairments in their access to large museums compared to in situ authentic culture [2]. People with hearing loss of any age who know written language and use lip-reading to support their access to spoken language will in the future be an even larger group compared to sign language users as deaf people are now being fitted with cochlear implants to help improve their listening ability. Mather, Gregory, and Archbold [3] reported that 15 young people who had received sequential bilateral cochlear implants all found improvements in listening with the second implant, even those who did not carry on wearing it.
There are also an increasing number of elderly people who have difficulties in hearing as they were approximately $12.5 \%$ of the population in 2010 and the percentage is predicted to peak at $20 \%$ by 2025 [4].

In order to reduce discrimination in access to information in Thai local museums, technology developers need to produce technology solutions. There has, however, been no framework that has helped technology developers to consider all of the possible interactions that occur at the same time and in the same place although there have been projects concerned with how to use technology to support some of these interactions. Section II provides an example museum scenario illustrating the problems, section III reviews related frameworks, section IV explains the TEIF, section V explains the TEIF Method, section VI explains the experimental design, section VII shows the experimental and questionnaire results, and section VIII is a conclusion to the paper.

\section{EXAMPLE THAI MUSEUM SCENARIO}

The following scenario based on an existing Thailand Tourism Award winning [5] small local museum visited by the author is presented to show current problems which affect people with hearing impairments in their access to Thai culture and history in local museums.

"Suchat Trapsin, who is the owner of the museum, allocated some parts of his house to become the Museum of Folk Art and Shadow Puppets in order to preserve the culture of shadow puppetry by teaching the public more about it. However, there is no information provided in text format inside the museum because normally Suchat will explain the history and tradition behind shadow puppetry by talking with visitors. When people with a hearing impairment watch the shadow puppet show, they cannot hear the conversation clearly because of the background music and the shadow puppet show is also fairly dark with Suchat operating the puppets from behind a screen, which makes lip-reading very difficult for them. Moreover, the language, which is used in the show, is quite complicated, and therefore sometimes difficult to understand if hearing impairment at birth affected their learning of language."

Other scenarios that were also investigated involving hearing impaired people included a tutorial, GP Surgery and Segway Tour in Rome. 


\section{REVIEW OF RELATED FRAMEWORKS}

An extensive review of the literature found that there was no framework based on principles rather than just technology system architectures or solutions to guide developers in developing technology solutions for such face-to-face situations for people with hearing impairment, other disabilities or no disabilities that included one or more interaction types involving people, technology, and objects. The research challenges that were therefore identified were to develop a Technology Enhanced Interaction Framework (TEIF) and TEIF Method regarding disabled people interacting with people, technologies, and objects. As information and communication technology has become more important in society, many researchers have been concerned with how to use technology to support communication between people and improve interactions between people, technology, and people $[6 ; 7 ; 8 ; 9 ; 10 ; 11]$ or improve interactions between people, technology and objects [10]. The details of interaction frameworks are reviewed under the following two main category headings.

\section{A. Interaction Frameworks}

Sung et al. [10] proposed a framework for designing a mobile electronic guidebook for a history museum. An electronic guidebook was implemented and evaluated in comparison to a worksheet and visiting without any guidebook or worksheet. Users spent the most time with exhibits when using the electronic guidebook but there were no significant differences in the knowledge gained about exhibits. Their framework did not consider a scenario where an expert presented or explained the exhibits. Rukzio [9] presented a physical mobile interaction framework for using mobile devices as mediator for the interaction with a physical object and discussed its implementation. The interactions were Human-Computer, Human-Real World, Computer-Real World, and Computer-Computer. Dix's framework for Computer Supported Cooperative Work [6] seems to address some of the possible interactions but it misses out some important interactions in the same time and at the same place situations such as people using technology to interact with real objects. In Dix's framework, the participants communicate with other participants in what is called 'direct communication'. Furthermore, the participants also interact with artefacts (man-made technology tools) by 'controlling' or 'acting'. Sometimes an artefact is shared between the participants; in this case, the artefact is not only the subject of communication but can become a medium of communication, called 'feedthrough', as shown in Figure 1.

In communication about work and the artefacts of work, various means are used to refer to particular artefacts, and Dix terms this 'deixis'. Dix [7] discussed the application of his CSCW Framework to mobile devices and Dix [8] used his framework to investigate issues determining the viability of the World Wide Web as an infrastructure for cooperative work. Vyas et al. [11] explored the role of artefacts supporting mediated communication. They used the term 'artefacts' to refer to analogue and digital objects that can serve as a tool in artefact - mediated communication. They asked students of a master's course on Visual Design to come up with and use a communication metaphor in their concept design process. They noted the use of communication metaphors is culturally dependent. Gaines [12] observed that recommendations based on practical experience of single users operating standard workstations had little to offer developers of complex systems integrating complex behaviour of people and computers. To address this issue he presents a conceptual framework for person-computer interaction in complex systems based on an analysis of systems theory literature to derive design principles for person-computer interaction and a hierarchical model of person-computer systems. His model acknowledges a technological system's behaviour reflects the value systems and inter-personal attitudes of the system designer and so the same systems principles apply to the psychology, sociology, human-computer interaction, and computer-computer interaction.

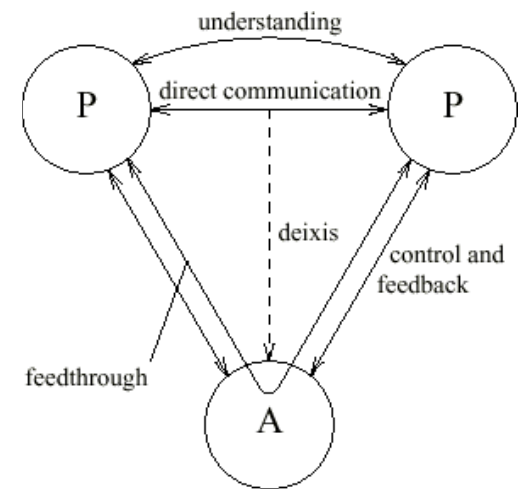

Fig. 1. Computer Supported Cooperative Work - A framework

\section{B. Considering Accessibility}

Cook and Hussey [14] modified Bailey's Human Performance Model in order to accommodate assistive technology. The components of their model are: human (abilities/skills), activity (determined by role), context (setting, social, cultural, physical), and assistive technology (hardware, software, non-electronic). The Lázaro Galdiano Museum [15] provides a Guide app with accessibility options including subtitling and video in Spanish Sign Language for the hearing impaired and audio description for people with visual disabilities. Features include zoom, high contrast and screen reader.

\section{TECHNOLOGY ENHANCED INTERACTION FRAMEWORK}

The TEIF that has been developed based on the main and sub-components identified in Table I and by adapting and extending the work of Dix [6] and Gaines [12] to help developers to design technology to support communication between people and improve interactions between people, technology and objects, particularly in complex situations involving disabled people. The TEIF aims to help developers design technology solutions to complex situations, particularly when disabled people are involved by helping developers think about the user requirements, designing interactions to meet these requirements and the criteria related to the requirements to evaluate the interactions. 
TABLE I TECHNOLOGY EHNANCED INTERACTION FRAMEWORK

\begin{tabular}{|c|c|c|}
\hline \multirow[t]{2}{*}{ Main Component } & \multicolumn{2}{|c|}{ Main and Sub-Component of Technology Enhanced Interaction Framework } \\
\hline & Sub-component & Explanations and Examples \\
\hline \multirow[t]{2}{*}{ People } & Role & $\begin{array}{l}\text { A person has a role when communicating with others (e.g. presenter, audience, peer). Roles } \\
\text { normally come in pairs such as speaker and audience (e.g. teacher and student or owner and } \\
\text { visitor) and peer to peer (e.g. student and student or visitor and visitor). }\end{array}$ \\
\hline & $\begin{array}{l}\text { Ability / } \\
\text { Disability }\end{array}$ & $\begin{array}{l}\text { People have abilities and disabilities which can affect their use of technology or } \\
\text { understanding of language and which can lead to communication breakdown (e.g. physical, } \\
\text { sensory, language, culture, communication, Information Technology (IT)). }\end{array}$ \\
\hline \multirow[t]{3}{*}{ Objects } & Dimension & $\begin{array}{l}\text { Objects have } 2 \text { dimensions (2D) or } 3 \text { dimensions (3D), and a 3D object may have a 2D } \\
\text { representation. }\end{array}$ \\
\hline & Property & Objects have colour, shape, size, and identity \\
\hline & Content & $\begin{array}{l}\text { Objects have content which is human readable (text, pictures) and machine readable (QR } \\
\text { code, AR tag, barcode, RFID tag, NFC). }\end{array}$ \\
\hline \multirow[t]{4}{*}{ Technology } & Electronic & $\begin{array}{l}\text { Electronic technology has stored information, is online (e.g. internet, phone network) or } \\
\text { offline (e.g. not connected to the internet or phone network), and is mobile (e.g. smartphone) } \\
\text { or non-mobile (e.g. desktop computer). }\end{array}$ \\
\hline & Usability and Accessibility & People interact with technology through its user interface (e.g. touch screen, keyboard). \\
\hline & $\begin{array}{l}\text { Application } \\
\text { or Service }\end{array}$ & Electronic technology is an application (e.g. dictionary) or a service (e.g. weather forecast). \\
\hline & Cost & Technology has cost (e.g. of hardware, software, maintenance). \\
\hline \multirow[t]{5}{*}{$\begin{array}{l}\text { Interactions and } \\
\text { Communication }\end{array}$} & $\begin{array}{l}\text { People-People } \\
\text { (P-P) }\end{array}$ & $\begin{array}{l}\text { People communicate verbally (speak, listen, ask, answer) and non-verbally (lip-read, smile, } \\
\text { touch, sign, gesture, nod). When communicating, people may refer (speak or point) to } \\
\text { particular objects or technology - this is known as 'deixis'. }\end{array}$ \\
\hline & People-Objects (P-O) & $\begin{array}{l}\text { People interact with objects for two main purposes: controlling (e.g. touch, hold or move), } \\
\text { and retrieving information (e.g. look, listen, read, in order to get information or construct } \\
\text { personal understanding and knowledge). }\end{array}$ \\
\hline & People-Technology (P-T) & $\begin{array}{l}\text { People control technology (e.g. hold, move, use, type, scan, make image, press, swipe), } \\
\text { transmit and store information (e.g. send, save, store, search, retrieve). }\end{array}$ \\
\hline & $\begin{array}{l}\text { People-Technology-People } \\
\text { (P-T-P) }\end{array}$ & $\begin{array}{l}\text { People use technology to transmit information to assist communication with (e.g. send SMS, } \\
\text { MMS, email, chat, instant message) other people. Technology is always designed for a } \\
\text { purpose by people and so a robotic device triggered by the person walking past it is a P-T-P } \\
\text { interaction. }\end{array}$ \\
\hline & $\begin{array}{l}\text { People-Technology } \\
\text {-Objects(P-T-O) }\end{array}$ & $\begin{array}{l}\text { People use technology (e.g. point, move, hold, scan QR codes, scan AR tag, use camera, use } \\
\text { compass) to transmit, store, and retrieve information (send, save, store, search, retrieve) to, in, } \\
\text { and from objects. }\end{array}$ \\
\hline \multirow[t]{2}{*}{ Time / Place } & Place & \multirow{2}{*}{$\begin{array}{l}\text { Same and different time and place yield four categories: same time (ST) and same place } \\
\text { (SP), different time (DT) and same place (SP), different time (DT) and different place (DP), } \\
\text { same time (ST) but different place (DP). }\end{array}$} \\
\hline & Time & \\
\hline \multirow[t]{6}{*}{ Context } & Location & $\begin{array}{l}\text { Location affects the use of technology (e.g. indoors, outdoors). For example GPS does not } \\
\text { work well indoors. }\end{array}$ \\
\hline & $\begin{array}{l}\text { Weather } \\
\text { Condition }\end{array}$ & $\begin{array}{l}\text { Weather condition may affect the use of technology (e.g. rainy, cloudy, sunny, windy, hot, } \\
\text { cold, dry, wet). For example, the mobile phone screen doesn't work well in sunshine. }\end{array}$ \\
\hline & $\begin{array}{l}\text { Signal Type } \\
\text { and Quality }\end{array}$ & Signal type can affect the quality of electronic technology (e.g. broadband, GPS, 3G, 4G). \\
\hline & $\begin{array}{l}\text { Background } \\
\text { Noise }\end{array}$ & $\begin{array}{l}\text { Background noise can affect the communication particularly for hearing impaired people (e.g. } \\
\text { background music, crowded situation). }\end{array}$ \\
\hline & Lighting & Light can affect the interaction (e.g. Inadequate light, too bright). \\
\hline & $\begin{array}{l}\text { Awareness of others' } \\
\text { interactions }\end{array}$ & People can be aware of interactions involving other people \\
\hline \multirow[t]{6}{*}{ Interaction Layer } & Culture & $\begin{array}{l}\text { Cultural layer includes countries, traditional, language and gesture (e.g. 'hello' is a normal } \\
\text { greeting used in the culture). }\end{array}$ \\
\hline & Intentionality & Intention layer involves understanding, purpose and benefit (e.g. the intent is a greeting). \\
\hline & Knowledge & $\begin{array}{l}\text { Knowledge layer involves facts, concepts, procedures, and principles (e.g. how to spell the } \\
\text { word 'hello'). }\end{array}$ \\
\hline & Action & $\begin{array}{l}\text { Action layer involves actions and behaviours (e.g. pressing the correct key and not hitting } \\
\text { neighbouring keys). }\end{array}$ \\
\hline & Expression & $\begin{array}{l}\text { Expression layer describes how actions are carried out (e.g. whether action is correct, } \\
\text { accurate, and prompt). }\end{array}$ \\
\hline & Physical & $\begin{array}{l}\text { Physical layer is the lowest layer at which people interact with the physical world (e.g. the } \\
\text { button is depressed and so sends the electronic code for the letter to the application). }\end{array}$ \\
\hline
\end{tabular}




\section{A. Terminology}

Terminology used in the TEIF is defined as follows:

- Communication is the process of passing information from one person to another [14].

- Technology is a tool that helps people achieve their purpose.

- People means anyone involved in direct communication or interaction with an object, technology, or other people.

- Object is not virtual any anything that is not a technology or a person involved in communication or interaction.

- Interactions can be between people and objects (P-O) or people and technology (P-T). People can also use technology to mediate interaction with people (P-T-P) or objects (P-T-O).

- A complex situation is a situation, which has the interaction in the forms of P-P, P-T, P-T-P, and P-T$\mathrm{O}$, especially when disabled people are involved.

\section{B. Main and Sub-components and Architecture of TEIF}

There are seven main components in the TEIF as shown in Table I. People can have roles, abilities, and disabilities. The components 'Object' and 'Technology' are used in order to extend Dix's framework to show any type of interaction and the overall architecture of the TEIF is shown in Figure 2. Objects are defined as having three sub-components: dimensions, properties, and content. Technology has a cost and can be electronic or non-electronic, online or off-line, and mobile or non-mobile. Furthermore, it may or may not have stored content and may additionally have an interface and be an application or provide a service. The TEIF involves interactions from P-P, P-O, P-T, P-T-P, and P-T-O. 'Time and Place' can be divided into four categories [15]. Context can include factors and constraints such as location, signal quality, background noise, lighting, and weather conditions. The TEIF layers and explanations have been adapted from Gaines' framework [12].

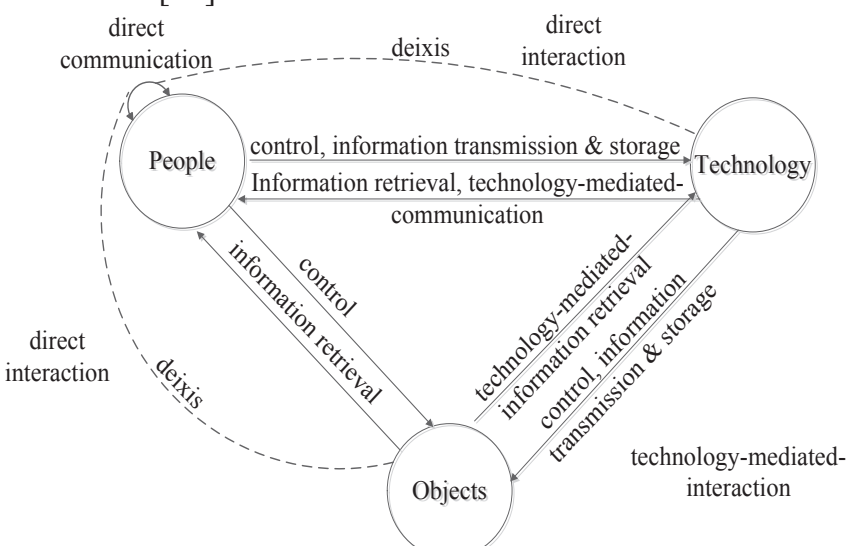

Fig. 2. Architecture of the Technology Enhanced Interaction Framework

$$
\text { V. TEIF METHOD }
$$

The TEIF Method has been developed in order to help developers understand and apply the TEIF to help make communication and interaction more accessible for people with disabilities. The TEIF Method does not replace other methods but supports them by providing multiple-choice questions to help identify requirements, the answers to which help provide technology suggestions that support the design stage. The TEIF Interaction Diagram and Use Case Diagram are also provided to help with this stage. The example scenario of a hearing impaired visitor to the Shadow Puppet Museum is also provided in section II to help understanding of the TEIF Method.

\section{A. TEIF Method Overview}

This section describes the steps used in the TEIF Method. There are main five steps in the TEIF Method. Developers can go through steps 3 to 5 as many times as required to develop the best solution.

Step 1: developers answer multiple-choice questions for gathering or evaluating requirements, which help identify any communication or interaction issues or problems involving hearing impaired people.

Step 2: developers use the technology suggestions table, which is based on answers to the multiple-choice questions, to help in designing or evaluating technology solutions.

Step 3: developers construct a technology solution scenario by using the technology suggestions table to help in designing or evaluating the solution.

Step 4: developers draw the TEIF overview Interaction Diagram showing the interactions between 'People', 'Technology', and 'Objects' to help in designing the technology solution and evaluating how technologies can be best combined.

Step 5: developers draw the TEIF overview Use Case Diagram to help in designing the technology solution, and evaluating how technologies can be best combined by showing the 'location' of interaction activities and the actions of the people and object 'actors' that are required to carry out the related tasks with the technology.

\section{B. Example Multiple Choice Questions for Requirements}

The TEIF Method helps developers gather or evaluate requirements by using multiple-choice questions, which developed, based on the TEIF. The questions help identify issues for which a technology solution is required. Four example questions are shown below:

12) What type of technology would be appropriate for the solution to the scenario?

a. online technology (Internet) b. off-line technology

c. I do not know

13) What type of technology devices would be appropriate for the solution to the scenario?

a. mobile devices b. non-mobile devices c. I don't know

16) Where does the situation take place?

a. indoors b. outdoors c. I do not know

17) What are the two main environmental considerations identified that impact the scenario?

a. noise (Background noise affects everyone's ability to hear 
and understand what is said)

b. room acoustics (surface (e.g. walls, windows, tile) and objects within every room interact to produce reverberation in response to sound.)

c. distance (How far is the audience standing from the presenter?)

d. visual access ( How well can the audience see everything that is happening in different locations?)

e. lighting (Inadequate lighting or large banks of windows can be challenging for deaf or hard of hearing audience because they cannot see the speakers face well or an interpreter may be located in shadows)

\section{Example Technology Suggestions Table}

The example technology suggestions table is based on a review of available technologies that can assist hearing impaired people and can be used to help identify one or more technologies than can be used for the solution by ensuring any combination of technologies used for the solution addresses all the requirement issues (i.e. all the issues have at least one tick in their column). For example, Suchat would like to use online technology indoors which is reflected in the answers to questions 12 and 16. He also would like to use Chuty's and her parents' smartphones to keep his costs low, which is shown in the answers to questions 13, 18, and 19. Many technologies may meet a requirement: for example, all of the technology suggestions shown in Table II. The technology suggestions in the table are listed in order of total score. The online version uses tooltips to display the explanations for the ticks or crosses.

\section{TABLE II TECHNOLOGY SUGGESSION TABLE}

\section{Example Technology Solution Scenario for Thai Local} Museum

To help developers understand how to use the technology suggestions, an example solution is provided that incorporates

\begin{tabular}{|c|c|c|c|c|c|c|c|c|c|}
\hline \multirow[b]{2}{*}{$\begin{array}{l}\text { Technology } \\
\text { suggestions }\end{array}$} & \multicolumn{9}{|c|}{ Which requirements the technology meets } \\
\hline & 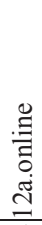 & 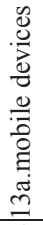 & 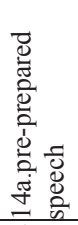 & 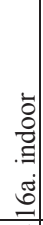 & 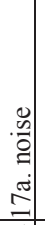 & 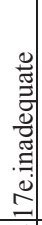 & $\begin{array}{c}\overrightarrow{0} \\
0 \\
0 \\
0 \\
0 \\
0.0 \\
0 \\
0\end{array}$ & 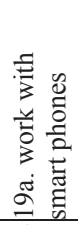 & 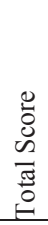 \\
\hline 1. Mobile web site & $\checkmark$ & $\checkmark$ & $\checkmark$ & $\checkmark$ & $\checkmark$ & $\checkmark$ & $\checkmark$ & $\checkmark$ & 16 \\
\hline $\begin{array}{l}\text { 2.Pre-prepared } \\
\text { caption/subtitle }\end{array}$ & $\checkmark$ & $\checkmark$ & $\checkmark$ & $\checkmark$ & $\checkmark$ & $\checkmark$ & $\checkmark$ & $\checkmark$ & 16 \\
\hline $\begin{array}{l}\text { 3. Frequently asked } \\
\text { questions (FAQ) }\end{array}$ & $\checkmark$ & $\checkmark$ & $\checkmark$ & $\checkmark$ & $\checkmark$ & $\checkmark$ & $\checkmark$ & $\checkmark$ & 16 \\
\hline 5. Instant messaging & $\checkmark$ & $\checkmark$ & $\checkmark$ & $\checkmark$ & $x$ & $\checkmark$ & $\checkmark$ & $\checkmark$ & 15 \\
\hline $\begin{array}{l}\text { 13. Speech } \\
\text { recognition }\end{array}$ & $x$ & $\checkmark$ & $\checkmark$ & $\checkmark$ & $\checkmark$ & $\checkmark$ & $\checkmark$ & $x$ & 13 \\
\hline
\end{tabular}

some of the technology suggestions shown in Table II.

'The technology developer has decided based on the TEIF Method's suggestions and discussions with their client Suchat to make available a mobile web site with which Chuty and her parents can use their smart phones to enhance the visit. There is a specific function called live functionality which assists
Chuty and her parents to communicate with Suchat. Chuty and her parents go to the mobile website and enable the function before the tour starts. The function allows Suchat to notify Chuty and her parents when the tour begins and change the topics by pressing the 'start' button on his mobile phone. Chuty and her parents' phones vibrate at the same time to notify them of this, and as Suchat starts speaking the preprepared summary captions for the first topic, appear on their smartphones. As the captions are presented on the mobile website, the words are highlighted in a sentence, allowing her to follow the conversation. Suchat can also notify Chuty and her parents that the topic is changing by sending a message to the server causing the captions to automatically change on the mobile website. Sometimes Chuty can't catch all of the conversation but she can pick out keywords through lip reading and hearing. Moreover, she can search for text by using automatic speech recognition of keywords, and then visually or manually select captions based on possible keywords which are highlighted in colour. So she can scroll up and down to find the conversation. When Suchat shows the shadow puppet, he uses his mobile phone to select and indicate the captions of the show that he is currently performing. Chuty and her parents then can enjoy the show by watching the shadow puppet and they also can read the captions when they need to on their smartphones. During the tour, Chuty asks some questions by typing instant messages on her smartphone which Suchat answers supported by selecting pre-prepared caption answers to frequently asked questions or by typing the answers on his smartphone. When Chuty asks questions about the exhibits, to help Chuty understand Suchat's answers she can use the further information displayed on her smartphone browser through the links from the QR codes on the exhibits.'

\section{EXPERIMENTAL DESIGN}

Following a successful expert review and validation of the TEIF and TEIF Method by three developer experts, three accessibility experts and an HCI professor, an experiment was carried out involving 36 experienced developers evaluating requirements and technology solutions. Half the participants used the TEIF Method and the other half used whatever other methods they would normally have used and were then shown the TEIF Method. The experiment was carried out in a face-toface situation with the researcher so the researcher was in control by passing the appropriate document to the participant and ensuring the participant engaged with the two experimental tasks and questionnaire. There was no significant difference between the TEIF Method and the Other Method groups in terms of the number of years of practical experience designing software and experience in designing technology solutions for disabled people and so the groups were well balanced in this respect. The participants in both groups were asked to select the best ten requirements from 29 possible requirements for a technology solution to the disability related problems they identified from the scenario interview transcript and underline the keywords of each answer on the transcript sheet. The participants were asked to give ratings between 0 
and 10 for how well each of the three solutions (A, B, C) provided met each of the ten requirements and were asked to underline the words on the solution sheets that helped them with their ratings. To assist them with this task they were provided with a modified version of the paper based technology suggestion table that was provided for the TEIF Method group that only contained descriptions of the technologies, without any ticks, crosses, or explanations. The participants were asked to give background information, rate the clarity of explanation of TEIF Method steps, evaluate whether and how the TEIF Method helped, imagine how the TEIF Method might help in the future, and any other comments about the usefulness or value of the TEIF Method.

\section{EXPERIMENTAL AND QUESTIONNAIRE RESULTS}

- The mean number of correct requirements was significantly higher $(\mathrm{p}<0.001)$ for participants using the TEIF Method $($ mean $=8.33)$ than the Other Methods (mean $=3.89$ ).

- $\quad$ The TEIF Method helped developers select a solution significantly $(\mathrm{p}=0.01)$ more often than the Other Methods that was not worse than a solution rated best by the experts.

- The TEIF Method helped developers rate solutions significantly closer $(\mathrm{p}<0.05)$ to the experts' ratings than the Other Methods for solution A but not for solutions B and $\mathrm{C}$, and this applied similarly to each requirement.

- The participants thought that the TEIF Method would also help with gathering requirements for technology solutions to interaction problems involving hearing impaired people, and for other disabled people.

- The participants thought that the TEIF Method would help designing technology solutions to interaction problems involving hearing impaired people, and if information was provided for other disabled people.

- $\quad$ Participants thought that the technology suggestions table in the TEIF Method helped with identifying technology solutions to interaction problems involving hearing impaired people.

- The participants thought that the TEIF Method would help designing technology solutions to interaction problems involving hearing impaired people, and if information was provided for other disabled people.

- Participants thought that the TEIF Method helped improve a developer's awareness of interaction issues involving hearing impaired people.

- Participants thought that the TEIF Method helped improve a developer's understanding of how environment context affects interaction involving hearing impaired people.

\section{CONCLUSION}

The TEIF and TEIF Method have been shown to have the potential to guide developers in developing technology accessibility solutions for Thai museums from the results of an experiment and questionnaire involving 36 experienced developers evaluating requirements and technology solutions. The TEIF has been developed based on the main and subcomponents and the TEIF Method had been developed to help developers to design technology to support communication between people and improve interactions between people, technology and objects, particularly in complex situations involving disabled people. Future work includes extending the requirement questions and technology suggestions tables to address the needs of people with a wider range of disabilities.

\section{REFERENCES}

[1] Princess Maha Chakri Sirindhorn Anthropology Centre. (2005). Local Museum Database Retrieved 07/02/, 2012, from $\mathrm{http} / /$ www.sac.or.th/databases/museumdatabase_eng/

[2] Proctor, N. (2005). Off Base or On Target? Pros and Cons of Wireless and Location-Aware Applications in the Museum Paper presented at the ICHIM 05 - Digital Culture \& Heritage, Paris, France. http://www.archimuse.com/publishing/ichim05/Proctor.pdf

[3] Mather, J., Gregory, S., \& Archbold, S. (2011). The Experiences of Deaf Young People with Sequential Bilateral Cochlear Implants Deafness \& Education International, 13(4), 152-172.

[4] National Electronics and Computer Technology Center, \& The National Telecommunications Commission. (2009). The provision of telecommunications services for the disabled and the elderly in Thailand. Bangkok, Thailand.

[5] ThailandTourist. (2014). Suchart Subsin, the Puppet Master and his Museum Retrieved 18/08/2014, 2014, from http://www.thailandtourist.net/guide/9_0_124_1_Puppet-Master-andMuseum.html

[6] Dix, A. (1994). Computer supported cooperative work - a framework. In Design Issues in CSCW Eds. D. Rosenburg and C. Hutchison. , Springer Verlag, 23-37.

[7] Dix, A. (1995). Cooperation without (reliable) communication: Interfaces for mobile applications. Distributed Systems Engineering, 2(3), 171

[8] Dix, A. (1997). Challenges for Cooperative Work on the Web: An Analytical Approach. Computer Supported Cooperative Work (CSCW) 6(2-3), 135-156. doi: 10.1023/a:1008635907287

[9] Rukzio, E. a. B., Gregor and Wetzstein, Sergej (2008). The Physica Mobile Interaction Framework (PMIF). Technical Report LMU-MI2008-2.

[10] Sung, Y.-T., Chang, K.-E., Hou, H.-T., \& Chen, P.-F. (2010). Designing an electronic guidebook for learning engagement in a museum of history. Computers in Human Behavior, 26(1), 74-83. doi: 10.1016/j.chb.2009.08.004

[11] Vyas, D., Dix, A., \& Nijholt, A. (2008). Role of Artefacts in Mediated Communication. Paper presented at the CHI 2008, Florence, Italy.

[12] Gaines, B. R. (1988). A conceptual framework for person-computer interaction in complex systems. Systems, Man and Cybernetics, IEEE Transactions on, 18(4), 532-541. doi: 10.1109/21.17371

[13] Cook, A., \& Hussey, S. (1995). Assistive Technologies: Principles and Pratice. Mosby: St. Louis, MO.

[14] Davis, K. (1977). Human Behavior At Work. New York: McGraw-Hill Book Co.

[15] The Lázaro Galdiano Museum launches the first application of the Áppside project. Available: http://www.gvam.es/fr/lazaro-galdianomuseum-launches-first-application-appsideproject/\#sthash.5NI5Iq9R.dpuf

[16] Ellis, J. R., Ridolfi, \& G. \& Zwirner, F. (1991). Groupware: Some issues and experiences. Communications of the ACM, 34(1) 